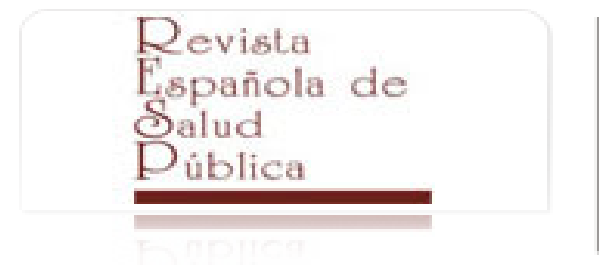

\title{
Revista Española de Salud Pública
}

ISSN: $1135-5727$

resp@msc.es

Ministerio de Sanidad, Servicios Sociales e

Igualdad

España

Jarillo Soto, Edgar C; Delgadillo Gutiérrez, Héctor Javier; Granados Cosme, José Arturo Conocimientos sobre el sida entre los estudiantes de medicina de tres universidades mejicanas

Revista Española de Salud Pública, vol. 74, núm. 2, marzo-abril, 2000, pp. 177- 187

Ministerio de Sanidad, Servicios Sociales e Igualdad

Madrid, España

Disponible en: http://www.redalyc.org/articulo.oa?id=17074208

- Cómo citar el artículo

- Número completo

- Más información del artículo

Página de la revista en redalyc.org

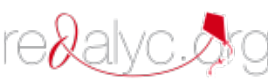

Sistema de Información Científica

Red de Revistas Científicas de América Latina, el Caribe, España y Portugal Proyecto académico sin fines de lucro, desarrollado bajo la iniciativa de acceso abierto 


\section{ORIGINAL}

\section{CONOCIMIENTOS SOBRE EL SIDA ENTRE LOS ESTUDIANTES DE MEDICINA DE TRES UNIVERSIDADES MEJICANAS}

Edgar C. Jarillo Soto, Héctor Javier Delgadillo Gutiérrez y José Arturo Granados Cosme

Universidad Autónoma Metropolitana-Xochimilco. México.

\section{RESUMEN}

Fundamento: Se considera el modelo médico hegemónico y se le articula con los postulados de la sociología de las profesiones. La información tiene al sida como tema concreto, porque presenta características de avance científico reciente, de importancia clínica y epidemiológica y por la investigación que se realiza para su tratamiento y la producción de vacunas.

Método: Es un estudio con estudiantes de distintos cursos de la carrera de medicina de las tres principales universidades del país. Se aplicó un cuestionario con preguntas cerradas que se probó y corrigió; las variables se refieren a tres tipos de conocimiento: básico, técnico y general.

Resultados: Entre los 120 estudiantes que respondieron al cuestionario hubo predominio del tercer $(30 \%)$ y cuarto $(32 \%)$ años de estudios. El $54 \%$ eran del sexo femenino. El primer año de estudios, con un intervalo de confianza del $95 \%$, presenta diferencias significativas $(p<0.05)$ con respecto de los estudiantes de $3^{\circ}$ y $4^{\circ}$ años de estudio. Al considerar la categorización por tipo de conocimiento se encontró que el conocimiento técnico tuvo mayor porcentaje de aciertos $(51 \%)$, seguido del conocimiento básico y el conocimiento general. El total de las respuestas a las 16 preguntas alcanzó una media de $42.7 \%$ de aciertos.

Conclusiones: Mostrando poco dominio sobre el tema, los conocimientos técnicos, resolutivos, de aplicación clínica, predominan sobre los sistemáticos, abstractos, del conocimiento general de la medicina. Se presenta una clara noción de los procesos en base al modelo médico hegemónico, en las relaciones unicausales de la enfermedad. Existe una extensión de los conocimientos básicos a la resolución de problemas clínicos. Los estudiantes de medicina se orientan a la asimilación de conocimientos que les resulten útiles en la práctica clínica

Palabras clave: Estudiantes de medicina. Formación profesional. Sida.

Correspondencia:

Edgar C. Jarillo Soto

Dirección personal:

Tejocotes 42-502

Col. Del Valle

03100, D.F. México

correo electrónico: jsec6322@ cueyatl.uam.mx

\section{ABSTRACT}

\section{Knowledge on Aids in the Medicine Student from Three University of Mexico}

Background: The Hegemonic Medical Model is discussed and is articulated with the postulates of the sociology of the professions. The information takes Aids as a specific topic, because it involves the aspects of recent scientific development, of clinical and epidemiological importance and due to the research which is being done for its treatment and the production of immunizations.

Method: This is a case study of students enrolled in different years of study for medical degrees at the three main universities in México City. A closed-question questionnaire was handed out, checked and corrected, the variables of which were related to three types of knowledge, that is, basic, technical and general.

Results: The students were revealed to master this subject to only a minor extent the extentions of the knowledge of the disciplines of the profession however being applied to new cases without the suitable accuracy. A certain degree of dissociation was found to exist among the different types of knowledge which were researched, the professional know-how having been noted to clearly conform within specialized, segmented, curative logic of the Hegemonic Medical Model.

Conclusions: The technical, problem-solving knowledge employed in clinical use prevails over the systematic. abstract knowledge of the general knowledge of medicine. A clear notion of the processes based on the dominan medical pattern is revealed in the single-causal relations of the disease. There is an extension of the basic knowledge a the resolving of clinical problems. The medical students are oriented toward taking in knowledge which will be useful to them in clinical practice.

Key words: Medical students. Professional knowledge. Ideology. Aids. 


\section{INTRODUCCIÓN}

Para identificar los procesos de construcción y los cambios del conocimiento científico en un área específica se puede partir del concepto de paradigma, porque permite distinguir su estructura, la cual coincide con el conocimiento específico que detentan las distintas profesiones ${ }^{1}$. En la profesión médica, además del paradigma científico está presente el atributo de profesión $\operatorname{docta}^{2}$.

Como parte de los rasgos profesionales está la formación universitaria, en la que los estudiantes de medicina adquieren los referentes técnicos y el conocimiento científico de la profesión ${ }^{3}$. La construcción de conocimiento técnico, especializado, docto y las experiencias educativas ocurren en el ámbito universitario y otras en las instituciones de salud y especialmente el hospital. En términos generales el conocimiento puede diferenciarse por niveles: en un extremo está el científico y en el otro el lego. Por lo tanto es posible identificar de modo simultáneo nociones científicas claramente estructuradas junto a nociones derivadas de la experiencia cotidiana, casi un conocimiento experiencial, empírico. Y una tercera categoría referida a nociones consecuencia de la extensión conceptual de nociones preconstruidas o inferidas a partir de conocimientos preexistentes $^{4,5}$.

La medicina presenta varios atributos que permiten caracterizarla: noción mecanicista de los procesos que estudia ${ }^{6}$, enfoque biologicista, orientación al individuo, con fuerte tendencia a la especialización, con progresiva incorporación y uso de la alta tecnología, énfasis en lo curativo y excluyente de otras formas de práctica médica ${ }^{7}$. Esa caracterización se complementa con la visión de la sociología de las profesiones, señala que el reconocimiento de la medicina como práctica curativa, se debe más a las habilidades técnicas del clínico y no tanto a los conocimientos «básicos» que detente.
Por lo tanto el paradigma de la medicina dominante presenta al menos tres tipos de conocimiento. El primero, que aquí se denomina conocimiento básico, relacionado con todo el conjunto científico de la profesión. El segundo, conocimiento técnico, se deriva del anterior; se construye por la práctica clínica y puede, aunque no necesariamente, tener un sustento sólido en el primer tipo de conocimiento. Por último, el conocimiento general de índole contextual, son nociones importantes para la profesión y también pueden ser necesarias y útiles para otras profesiones o prácticas, circula ampliamente en los medios de divulgación y no presenta mucha especificidad.

La transmisión, circulación y adopción del conocimiento médico no son circunstancias exclusivas ni circunscritas al espacio educativo, son influidas por el espacio de práctica hospitalaria y por el acceso prácticamente indiscriminado a medios masivos de divulgación y especializados de la medicina. Adquieren rigor en el espacio educativo y hospitalario, a través de conferencias, libros, revistas técnicas, clases formales, revisión de casos clínicos, reflexión de acciones diagnósticas o terapéuticas. En los estudiantes de medicina una forma peculiar de obtención de conocimientos se logra por la resolución de problemas en la práctica clínica, no necesariamente con racionalidad científica; las influencias publicitarias de los laboratorios, y por supuesto el acceso abierto y diverso a revistas de divulgación.

Para aproximarse al conocimiento y al paradigma que sustentan los estudiantes de medicina se consideró el sida, porque es un tema de actualidad que plantea muchos retos al conocimiento científico. Se parte del supuesto de que para acceder al nivel de conocimiento especializado, es necesario operar sobre bases propias de algunas disciplinas que forman parte de la medicina como son: inmunología, microbiología, virología. El sida es una enfermedad que en sí misma presenta un reto profesional para su diagnóstico, control y eventual tratamien- 
to; además, sus características epidemiológicas implican un tránsito constante entre lo asentado científicamente y lo supuesto empíricamente. Diversos indicadores permiten explorar los distintos niveles del paradigma científico de la profesión tomando como modelo al sida.

\section{SUJETOS Y MÉTODO}

Se diseñó un cuestionario considerando las variables e indicadores con preguntas de opción múltiple (Anexo 1). Las preguntas fueron formuladas con base en los contenidos comunes de los diferentes planes de estudios de cada institución. Para definir los parámetros de respuestas correctas e incorrectas, se construyó un patrón completo de aciertos, estructurados a partir de la revisión de textos técnicos ${ }^{8}$, artículos técnicos 9 , revistas especializadas, libros ${ }^{10,11}$ y folletos de divulgación ${ }^{12}$.

Se realizó una prueba piloto con dos muestras de estudiantes de medicina de una institución que no se incluyó en el estudio. Como resultado de la primera prueba se hicieron dos correcciones. La primera permitió identificar preguntas con un índice muy alto de no respuesta, por lo cual se excluyeron dos y se corrigieron tres preguntas formulándose de modo más simple. La segunda prueba sirvió para estimar el modo de codificar las preguntas e introducir más alternativas y combinaciones de respuestas.

Se estableció un criterio de selección no probabilístico y se definió una cuota de muestreo de 120 sujetos. La selección de la muestra fue por conglomerados, uno para cada escuela de medicina de las tres principales instituciones públicas de educación superior de la capital del país. De entre ellos se diseñó una selección aleatoria de acuerdo a las listas de cada grupo de alumnos. Se mantuvo la proporción de estudiantes según el grado de estudios. La muestra se constituyó con cuarenta estudiantes de medicina de cada institución. La aplicación de los cuestionarios se realizó entregándole a cada estudiante un modelo impreso y dejando la libertad de responder. A ningún estudiante de los 19 que formularon preguntas se les brindó información adicional.

El cuestionario se codificó con base a los parámetros establecidos y se les otorgaron opciones categóricas, así cada pregunta se cuantificó con valor de 1 para las correctas y 0 para las erróneas. Se utilizó la base de datos Excel 7.0. Los datos se exportaron al paquete estadístico SPSS versión 8.0. Los cálculos estadísticos se hicieron considerando las categorías de conocimientos científico, técnico y general; las diferencias entre ellos se contrastaron por el análisis de varianza de una vía o de un criterio de clasificación. Se analizó la interacción entre el nivel de estudios y las categorías del conocimiento a través del análisis de varianza para un factorial. Los factores fueron dos: nivel de estudios y tipo de conocimiento. El nivel de significancia fue de 0.05 , por lo que se tomaron como estadísticamente significativos los valores de $\mathrm{p}<0.05$.

\section{RESULTADOS}

En los 120 estudiantes que respondieron al cuestionario hubo predominio del tercer (30\%) y cuarto (32\%) años de estudios, el $54 \%$ eran del sexo femenino. En el cuestionario final no se obtuvo ninguna respuesta acertada a la pregunta 7 (¿identifica qué es el sitio AL-121?) por lo cual se decidió excluirla para el análisis estadístico. Asociando el nivel de estudios con las respuestas correctas (figura 1) se presenta una curva ascendente de los primeros años a los superiores, con un leve declive en el último año de estudios. La dispersión de los aciertos de estudiantes de primero y quinto años es mayor respecto de los niveles intermedios, en estos últimos los aciertos presentan una respuesta de modo más concentrado hacia la media. El primer año de estudios, con un 
Edgar C Jarillo Soto et al

Figura 1

Media y dispersión de aciertos a todas las preguntas según grado. Estudiantes de medicina México, D.F. 1997

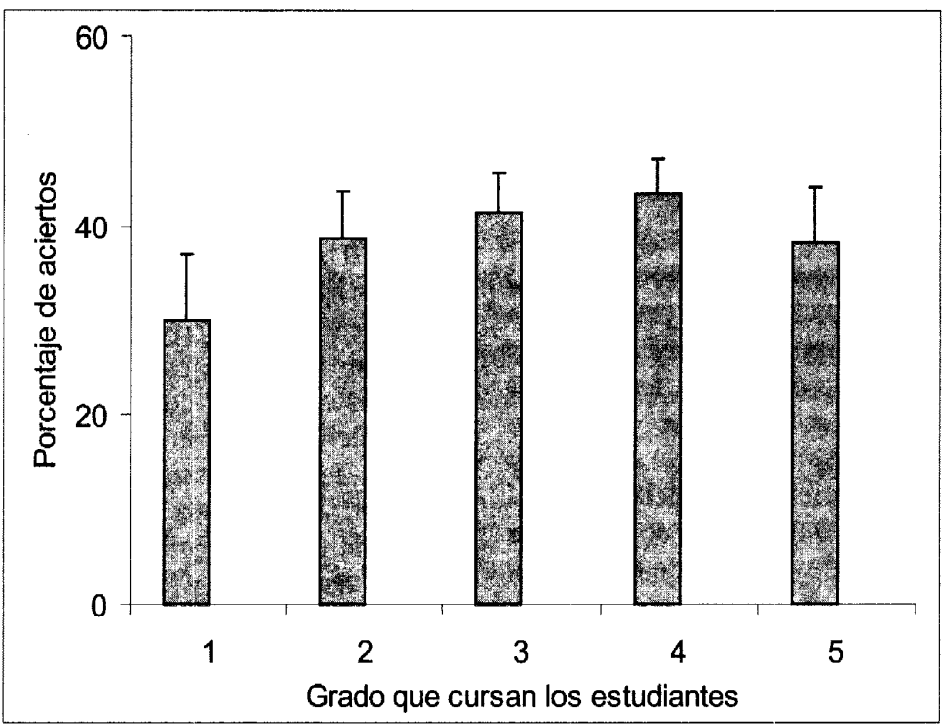

Figura 2

Respuestas correctas por tipo de conocimiento. Estudiantes de medicina. México D.F. 1997

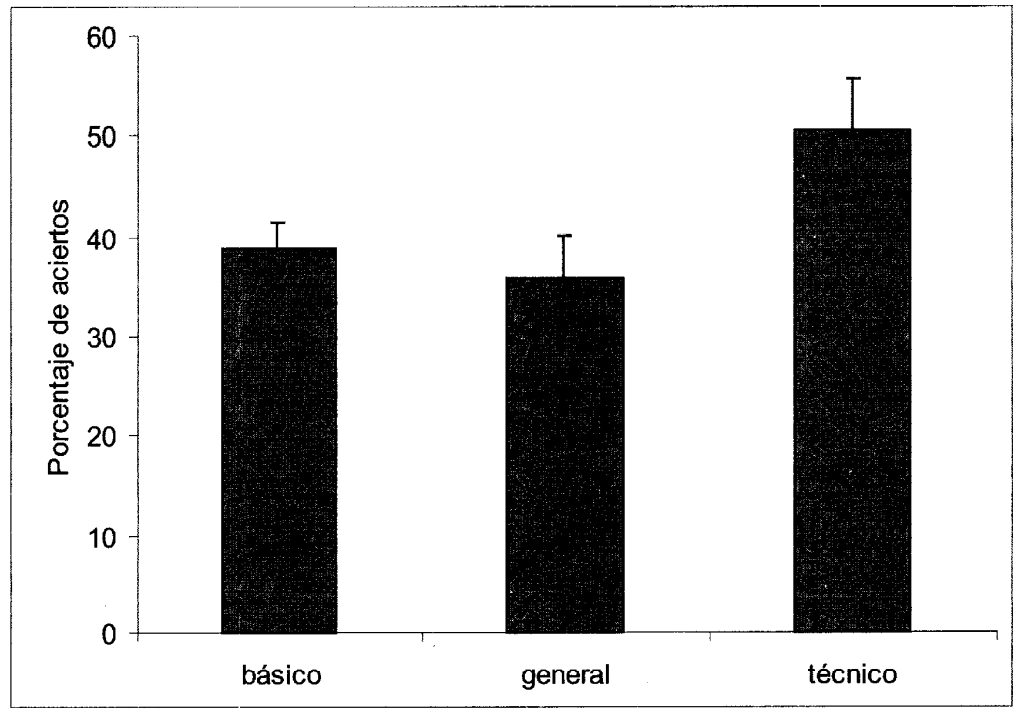


intervalo de confianza del $95 \%$, presenta diferencias significativas $(\mathrm{p}<0.05)$ con respecto de los estudiantes de $3^{\circ}$ y $4^{\circ}$ años de estudio.

Al considerar la categorización por tipo de conocimiento (figura 2) se encontró que el conocimiento técnico tuvo mayor porcentaje de aciertos $(51 \%)$, seguido del conocimiento básico y el conocimiento general. El conocimiento básico tiene menor dispersión y por lo tanto una consistencia más alta que los otros dos tipos de conocimientos. $\mathrm{Al}$ combinar las tres categorías con un nivel de confianza de $95 \%$ se manifestó una diferencia entre ellas estadísticamente significativa, con una $\mathrm{p}<0.05$. Pero aún más, el conocimiento técnico con una $\mathrm{p}<0.001$ tuvo una diferencia significativamente más alta respecto de los conocimientos básico y general.

El total de las respuestas (figura 3) a las 16 preguntas alcanzó una media de $42.7 \%$ de aciertos. No obstante esa concentración, cada una de las diferentes preguntas obtuvo medias muy variables. En el conjunto más alto están preguntas relativas a: dificultades para el desarrollo de una vacuna (9), familia de pertenencia del VIH-2 (10), sitio de detección del antígeno p24 (13), la prueba confirmatoria de infección (14), así como los países que realizan investigación para el desarrollo de una vacuna (17). En el rango intermedio cercano a la media general están: líneas actuales de investigación (16) y efectos colaterales en la administración del AZT (5). Por último, las tres preguntas con aciertos más bajos fueron: diferencias entre VIH1 y VIH-2 (2), asociación del AZT con otros fármacos para el control de la infección (6) y las investigaciones actuales para el desarrollo de una vacuna (15).

La asociación de los distintos grados que se cursan y las respuestas correctas fue muy directa para el conocimiento básico, especialmente para los niveles $3^{\circ}$ y $4^{\circ}$, siendo la diferencia con los demás niveles estadísticamente significativa $(\mathrm{p}<0.037)$. Para este tipo de conocimiento las preguntas sobre las célu-

Figura 3

Media y dispersión de cada pregunta. Estudiantes de medicina. México, D.F. 1997

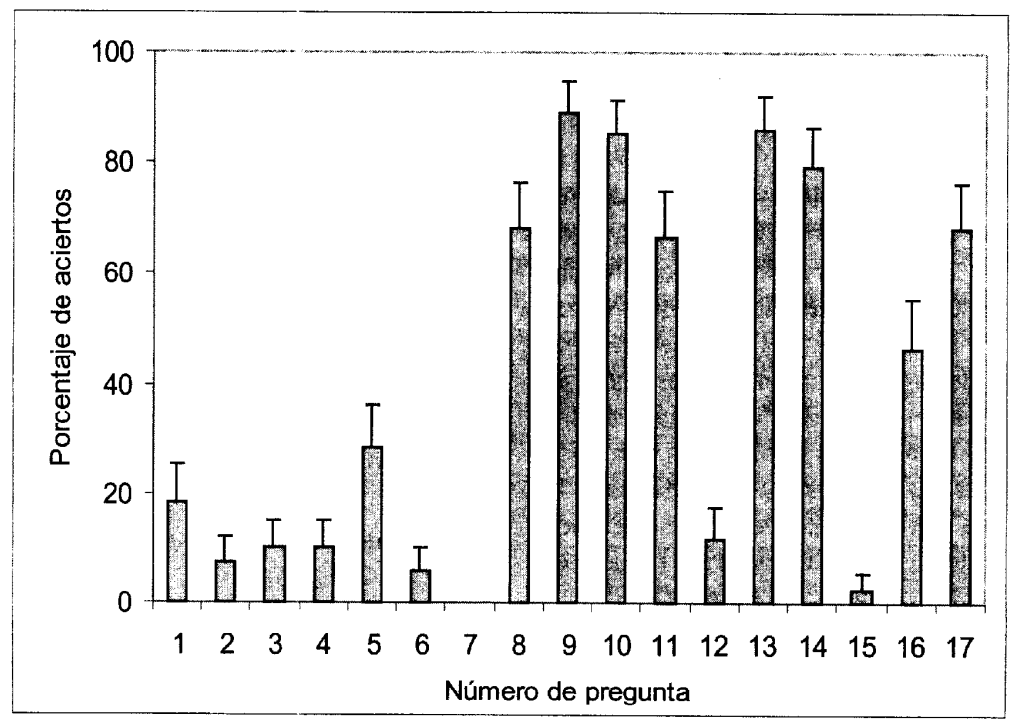

Rev Esp Salud Pública 2000, Vol. 74, N. ${ }^{\circ} 2$ 
Figura 4

Indicador características del agente, según grado del curso. Estudiantes de medicina. México, D.F. 1997

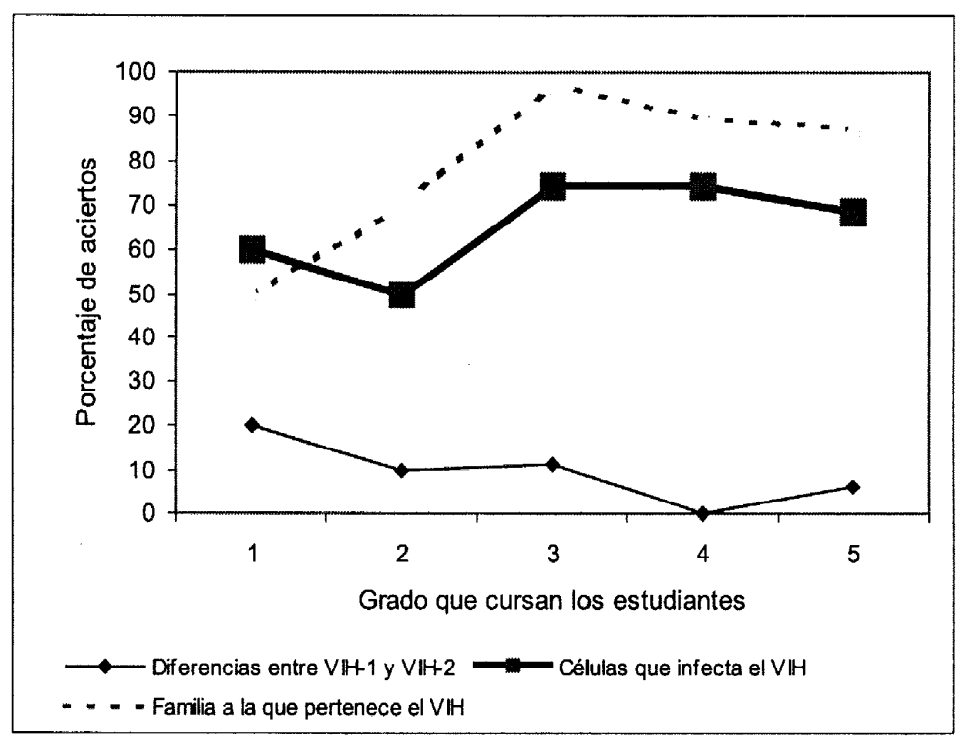

las que infecta el VIH (8) y la familia de pertenencia del virus (10), fueron las de mayor asociación entre el nivel de estudios y el conocimiento técnico. Con independencia del grado de estudios se encontraron diferencias estadísticamente significativas entre las distintas preguntas que integran este indicador. Así, con la prueba de Tukey para comparaciones múltiples, se encontró una $\mathrm{p}<0.001$ entre las preguntas 6,11 y 14 .

El conocimiento básico ocupa la segunda posición por su porcentaje de respuestas correctas, a su interior los distintos indicadores específicos presentan algunas variaciones. En lo referido a las bases microbiológicas del agente, las preguntas sobre la familia de pertenencia del VIH y las células que infecta, alcanzaron promedios cercanos al $100 \%$ para la primera y de $70 \%$ para la segunda, en ambos casos durante los años superiores de la carrera (Figura 4). La pregunta sobre las diferencias entre VIH 1 y 2 , presenta su valor más alto (20\%) durante el primer año de estudios y desciende a casi $0 \%$ en el cuarto año.
En el caso de la infección por VIH se requieren pruebas de laboratorio, de diagnóstico inicial y confirmatorias. Por ello se planteó un indicador con dos preguntas: la sensibilidad de la prueba de ELISA en el diagnóstico, especialmente en los falsos positivos y el conocimiento del sitio de detección para la P24 del VIH que implica bases virológicas relacionadas con el conocimiento básico. Los resultados de ese indicador (figura 5) muestran un porcentaje de respuestas acertadas para el sitio de detección de la P24 cercano al $100 \%$ en el primer año de la carrera, el cual decrece progresivamente según aumentan los años de estudio que se cursan. Un comportamiento de respuesta distinto lo presenta la pregunta sobre la sensibilidad de la prueba de ELISA, allí el valor más alto de aciertos no es superior al $20 \%$ con un leve descenso en el $4^{\circ}$ año de estudios, lo que se corrige con el ajuste de la curva.

Para el indicador fármacos de control para la infección por VIH (figura 6), se distinguieron dos preguntas: una dirigida a las 
CONOCIMIENTOS SOBRE EL SIDA ENTRE LOS ESTUDIANTES DE MEDICINA...

Figura 5

Indicador diagnóstico de infección, según grado del curso. Estudiantes de medicina. México, D.F. 1997

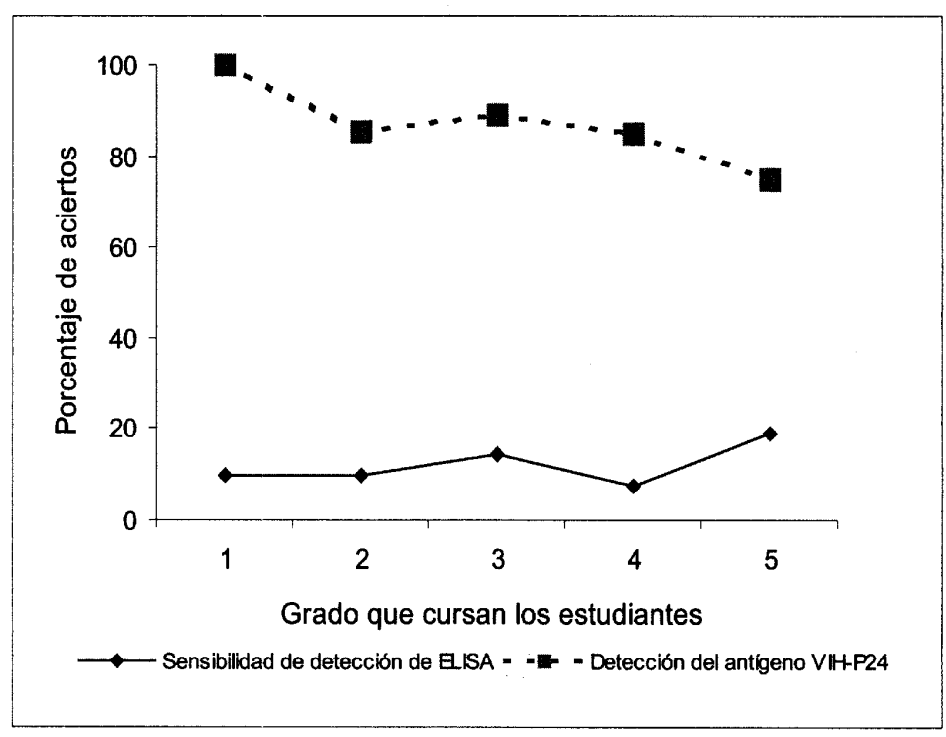

Figura 6

Indicador fármacos de control, según grado que cursan. Estudiantes de medicina. México D.F. 1997

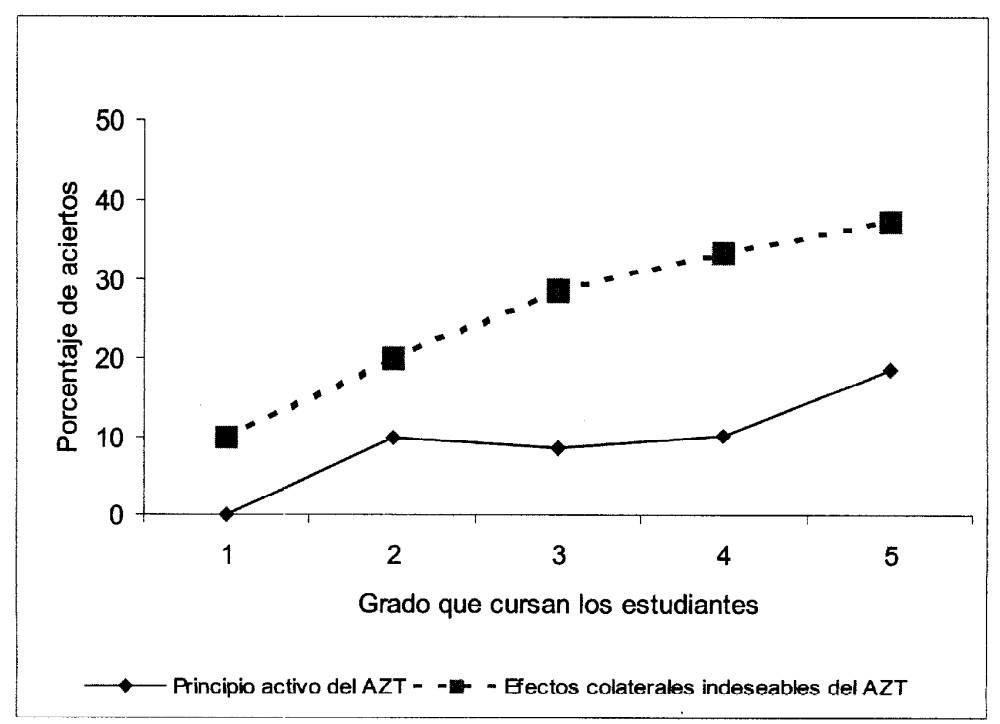

Rev Esp Salud Pública 2000, Vol. 74, N. ${ }^{\circ} 2$ 
bases de la farmacología, como es el principio activo del AZT. El nombre técnico de estc producto obtuvo valores de aciertos inferiores al 20\%; con un incremento progresivo conforme los años de la carrera son superiores. La otra pregunta sobre la identificación de algunos efectos colaterales en la administración prolongada del AZT, muestra una tendencia ascendente de la recta según se avanza en los años de la carrera, las respuestas con valores bajos en el año inicial se incrementan casi tres veces al último año del ciclo académico.

Las curvas de las tres gráficas anteriores manifiestan una constante: las respuestas con mayor porcentaje de aciertos están más asociadas a los conocimientos básicos que se imparten en los primeros años de la carrera, las respuestas acertadas referidas a conocimientos de la práctica profesional alcanzan valores más altos en los últimos años de la carrera.

\section{DISCUSIÓN}

Los resultados muestran que los alumnos de la población estudiada obtuvieron mayores calificaciones en el bloque de conocimiento técnico, mientras que lo referido al conocimiento general presenta las puntuaciones más bajas. Este hallazgo puede interpretarse como un predominio en el manejo de aspectos relacionados con la práctica clínica y cierta desarticulación entre el conocimiento básico y el conocimiento general, son los que se imparten en las aulas universitarias.

En nuestros resultados se observa una «disociación» del conocimiento médico. Por un lado el conocimiento básico que da fundamento y es un soporte del prestigio de la medicina; por otro el conocimiento técnico que es el que se asocia a la resolución de problemas de salud. Por lo tanto muestra una aparente discriminación de saberes con base en el sentido práctico y resolutivo de la acción técnica, que se requiere en la práctica profesional de manera directa. El predominio de lo resolutivo inmediato y práctico en detrimento de lo sistemático, abstracto y no directo. Rosenthal y Ogden ${ }^{13}$ en un estudio sobre las creencias de los estudiantes de medicina acerca de la educación médica, encontraron que la orientación científica de los alumnos disminuye gradualmente conforme avanzan los años de la escolaridad. Esos autores basan la reducción del interés por la actividad científica en la percepción de los alumnos dada la poca utilidad de las ciencias básicas con relación a su práctica clínica

Lo anterior se corrobora porque el indicador «características del agente» presenta algunas diferencias dignas de evidenciar. Las respuestas correctas son mayores respecto a las células que infecta el VIH y a la familia a la que pertenece el virus, mientras que mostraron menor acierto para las diferencias entre los tipos de VIH y sobre el punto de identificación AL-721 del virus. En la práctica médica durante el ejercicio cotidiano de la medicina, es fundamental la habilidad para la precisión del diagnóstico, la prescripción del tratamiento correspondiente y la verificación de su eficacia. En este sentido el conocimiento de las respuestas correctas a las dos primeras preguntas forma parte de la información que se difunde en los medios masivos de información y parece que para los estudiantes tiene mayor utilidad en términos de resolución de problemas prácticos. Así por ejemplo, uno de los indicadores usados tanto para el diagnóstico como para el seguimiento de la eficiencia de la terapéutica es precisamente el conteo de linfocitos CD4, por lo cual se revela ese aspecto con mayor importancia que la diferenciación de las características entre tipos de VIH, por más que sean relevantes para identificar la infección.

Por otro lado, ese rasgo también se corresponde con el modelo médico hegemónico, ya que prevalece por una parte el uso de alta tecnología y por otro el ejercicio clínico 
basado en relaciones de causa-efecto para derivar la terapéutica con énfasis en el aspecto curativo. En esa lógica, ante una impresión diagnóstica del médico el siguiente paso es la confirmación por medio del uso de la tecnología, para ello requiere conocer la prueba de laboratorio a solicitar, así como la forma de interpretar sus resultados; de ahí que en términos de eficacia sea necesario que los futuros profesionales conozcan correctamente las pruebas confirmatorias y las células blanco que ataca el VIH cuyo número puede ser reportado en pruebas de laboratorio.

El enfoque de causa-efecto de la enfermedad y su solución a través del énfasis curativo hace que las nociones de conocimientos científicos se expresen en el pensamiento médico de una forma peculiar, ya que ante un diagnóstico cualquiera se identifica el fármaco a prescribir en función de la eliminación del agente causal, tomando el resto de medidas terapéuticas (dietas, ejercicio físico e incluso fármacos no centrales) como elementos complementarios y accesorios. Este paradigma explica que los alumnos conozcan la toxicidad del fármaco central (AZT) pero no logren identificar con exactitud los medicamentos con los que éste debe administrarse.

Al considerar los modelos biologizados, curativos, individuales, como expresión del conocimiento profesional, se puede derivar que al mismo tiempo que se convierten en el núcleo duro de las ciencias médicas ${ }^{14-16}$, también constituyen un reforzamiento al justificar su erudición en las bases científicas de disciplinas reconocidas. Pero si lo específico es resultado de la asimilación y replicación de conocimientos, entonces su adquisición requiere de un proceso de formación especializada y por lo tanto la necesidad de una formación escolarizada de muchos años.

El ejercicio profesional se constituye en un valor que no es ajeno a los determinantes más generales de la sociedad, pero en térmi- $s$

nos técnicos en la profesión se reconoce que la atención de la enfermedad es lo fundamental. Así, diagnosticar correctamente y curar efectivamente es lo específico de la profesión. Y parece que a pesar de las menciones al humanismo y visión universalista de los profesionales de la medicina ${ }^{17}$ al menos para esta muestra, no se corresponde de manera clara. Esto se nota en los elementos contextuales del conocimiento científico tales como personajes identificados por sus aportaciones al avance científico, fechas cuando se ubican avances importantes. Muchos de estos datos no sólo circulan en las fuentes especializadas sino además en los medios masivos de comunicación y por su trascendencia para el SIDA, debieran ser conocidos muy bien por los estudiantes de medicina.

Otro aspecto relevante se refiere a las nociones que se articulan con el conocimiento científico. Así por ejemplo Kopacz y otros $^{18}$ diseñaron un estudio para valorar la actitud de los estudiantes ante el SIDA. La misma intención se exploró en otros trabajos ${ }^{19-21}$. En ellos se encontró que los estudiantes antes de actuar en función de sus conocimientos científicos, se basan en prejuicios para establecer un diagnóstico e indicar cierta terapéutica. Ello implica que la precisión del núcleo duro del conocimiento se hace extensivo a problemas técnicos que se presentan en la práctica profesional. Algo similar se observa en nuestros datos ya que se hacen extensivos los conocimientos básicos con base muy general a los conocimientos técnicos de aplicación en la práctica profesional.

Las respuestas acertadas que dieron los estudiantes evidencian poca importancia para el conocimiento general. Puede concluirse que este conocimiento para ellos no tiene influencia directa en la eficacia de los diagnósticos y tratamientos que lleguen a prescribir. Por lo tanto es una información prescindible e irrelevante, mucho más por lo que se expresa como disociación del conocimiento teórico y del conocimiento profesional. 
De forma general las características de las respuestas acertadas ofrecen la suposición de que los estudiantes portan nociones preconcebidas acerca de lo que representa la práctica médica y en función de ellas marcan limites a su interés por la obtención de conocimientos, por lo tanto es coherente que se orienten más a los la información y conocimientos que les brinden mejores posibilidades resolutivas.
Mientras que, por ejemplo, la esfera de la investigación puede resultarles menos atractiva como práctica profesional o como área de conocimiento. Esto es congruente con la falta de aciertos sobre las principales líneas de investigación en torno a la obtención de alguna vacuna, tanto para la trascendencia de la investigación básica como por las implicaciones para el conocimiento general.

Anexo 1

Preguntas del cuestionario, según indicador y tipo de conocimiento

\begin{tabular}{|c|c|c|c|}
\hline Tipo de conocimiento & Indicadores & $\begin{array}{l}N^{v} \text { de pregunta } \\
\text { en el cuestionario }\end{array}$ & Pregunta \\
\hline \multirow[t]{10}{*}{ BASICO } & \multirow{4}{*}{ Características del agente } & 2 & Diferencias entre VIH-I y VIH-II \\
\hline & & 7 & Qué es el AL-121 \\
\hline & & 8 & Células que infecta el VIH \\
\hline & & 10 & Familia a la que pertenece el VIH \\
\hline & \multirow{2}{*}{ Diagnóstico de infección } & 12 & Sensibilidad de detección de ELISA \\
\hline & & 13 & Detección del antígeno VIH-P24 \\
\hline & \multirow{2}{*}{ Fármacos de control } & 4 & Principio activo del AZT \\
\hline & & 5 & Efectos colaterales indeseables del AZT \\
\hline & \multirow[t]{2}{*}{ Desarrollo de vacuna } & 15 & $\begin{array}{l}\text { Investigaciones actuales sobre una } \\
\text { vacuna }\end{array}$ \\
\hline & & 9 & $\begin{array}{l}\text { Dificultades técnicas para desarrollar } \\
\text { una vacuna }\end{array}$ \\
\hline \multirow[t]{3}{*}{ TÉCNICO } & \multirow[t]{2}{*}{ Acciones de control } & 6 & Asociación del AZT con otros fármacos \\
\hline & & 11 & Toxicidad del AZT \\
\hline & $\begin{array}{l}\text { Confirmación de } \\
\text { diagnóstico }\end{array}$ & 14 & Prueba confirmatoria de infección \\
\hline \multirow{3}{*}{ GENERAL } & \multirow{2}{*}{ Información del Sida } & 1 & Descubridores del VIH-II \\
\hline & & 3 & Año de descubrimiento del VIH-II \\
\hline & $\begin{array}{l}\text { Conocimiento de } \\
\text { soluciones }\end{array}$ & $\begin{array}{l}16 \\
17\end{array}$ & $\begin{array}{l}\text { Líneas de investigación actuales } \\
\text { sobre SIDA } \\
\text { Países que desarrollan vacunas } \\
\text { contra el VIH-II }\end{array}$ \\
\hline
\end{tabular}




\section{BIBLIOGRAFÍA}

1. Kuhn TS. La estructura de las revoluciones científicas. México: Fondo de, Cultura Económica; 1972.

2. Friedson E. La profesión médica. Un estudio de Scociología del conocimiento aplicado. Barcelona: Península; 1978.

3. Rabkin M. A paradigm shift in academic medicine. Acad Med. 1998, 73:127-131.

4. Oriol A. Les competencies professionals en ciencies de la salut. Barcelona : IES; 1998.

5. Pereira A. Tornanr-se cientista: o ponto de vista de Bruno Latour. Cad Saúde Publ. 1997; 13:109-118.

6. Capra F. El punto crucial. Ciencia, sociedad y cultura naciente. Barcelona: Integral Editores; 1985.

7. Menéndez E. Introducción. En: Basaglia F, compilador. La salud de los trabajadores. Aportes para una política de la salud. México: Nueva Imagen;1978.p.11-53.

8. De Vita V. Sida. México: Salvat; 1995.

9. Polsky B. Treatment of HIV infections and its complications. Clin Chest Med, 1996;17:647663.

10. Dietterich H. Sida, cáncer, Parkinson. México: Planeta; 1997.

11. Montaigner L. De los virus y de los hombres. México: Océano; 1997.
12. Conasida. Folletos de divulgación. México: Conasida; 1996.

13. Rosenthal J, Ogden J. Changes in medical education: the beliefs of medical students. Med Educ. 1998;32:127-132.

14. Oriol A. Les competencies professionals en ciencies de la salut. Barcelona : IES; 1998.

15. Paris J. Investigación cualitativa en salud: superando los horrores metodológicos. En: Bronfman M y Castro R Coordinadores. Salud, cambio social y política. México: Edamex; 1999:35-48.

16. Lakatos I. La metodología de los programas de investigación científica. México: Alianza Editorial; 1983.

17. Chávez I. México en la cultura médica. México: Fondo de Cultura Económica; 1987.

18. Kopacz D. et al. Medical students and AIDS: knowledge, attuitudes and implications for education. Health Educ Res. 1998;14:1-6.

19. Mc Daniel J. A survey of knowledge and attitudes about HIV and AIDS among medical students. J Am Coll Health. 1995;44:11-14.

20. Zuber J. et al. Analysis of preconcevid attitudes of medical personnel toward HIV positive and AIDS patients. Psychother Psychosom Med Psychol. 1996;46:52-60.

21. Kelly J, Lawrence J, Smith S, Hodd H, Cook D. Medical student's attitudes towards AIDS and homosexual patients. J Med Educ 1987;62:549-556. 